\title{
Microbiological Safety and Antibiogram Analysis of Selected Food Products Obtained in the Marketplace of Peshawar and Mardan, KPK, Pakistan
}

\author{
Hayat Khan ${ }^{\# 1,2 *}$, Shakir Ullah"2, Muhammad Salman ${ }^{2}$, Firasat Hussain ${ }^{1,3 * *}$, \\ Yasir Anwar ${ }^{4}$, Ihsan Ullah ${ }^{4}$, Imtiaz Ali Khan ${ }^{5}$, Hazrat Hussain', \\ Ijaz Naeem ${ }^{6}$, Ajmal Khan ${ }^{7}$, Haji Khan ${ }^{8}$, Farooq Jan', Muhammad Shuaib ${ }^{10 * * *}$ \\ ${ }^{1}$ Department of Microbiology, University of Swabi, Pakistan \\ ${ }^{2}$ Department of Microbiology and Biotechnology, Abasyn University Peshawar, Pakistan \\ ${ }^{3}$ Department of Microbiology, Cholistan University of Veterinary \& Animal Sciences Bahawalpur 63100, Pakistan. \\ ${ }^{4}$ Department of Biological Sciences, Faculty of Science, King Abdulaziz University, Jeddah, Saudi Arabia \\ ${ }^{5}$ Department of Entomology, The University of Agriculture Peshawar, Pakistan \\ ${ }^{6}$ Department of Biotechnology, University of Swabi, Ambar 23561, Pakistan. \\ ${ }^{7}$ Department of Biotechnology, Bacha Khan University, Pakistan \\ ${ }^{8}$ Center of Biotechnology and Microbiology, University of Swat, Pakistan \\ ${ }^{9}$ Department of Botany, Abdulwali Khan University, Mardan, Pakistan \\ ${ }^{10}$ School of Ecology and Environmental Science, Yunnan University, Kunming, China
}

Received: 12 August 2018

Accepted: 8 September 2018

\begin{abstract}
Foodborne diseases are very frequent and can easily transmit from contaminated food and food handlers. Among the foodborne pathogens, strains of Staphylococcus aureus, Bacillus cereus, Listeria monocytogen and Clostridium are very important because of its presence in a wide range of foodstuffs. A total of 520 food samples were collected at Peshawar (50\%) and Mardan (50\%), Khyber Pakhtunkhwa, Pakistan, from April 2016 to March 2017. Isolates were cultured and discriminated by Gram stain, followed by biochemical identification, disk diffusion assay was performed using antibacterial and antifungal agents. Out of 520, only $122(23.46 \%)$ samples were positive for various types of bacterial and fungal pathogens. Gram-positive bacteria was the predominant $(70.49 \%)$ followed by fungal pathogens (26.23\%) and Gram-negative bacterial pathogens (3.27\%) among the total positive samples. Prominent bacterial samples were Staphylococcus aureus, Bacillus spp., Clostridium spp., Staphylococcus
\end{abstract}

\footnotetext{
\# Contributed equally

*e-mail: hayatkhan@uoswabi.edu.pk

**e-mail: firasat@uoswabi.edu.pk

***e-mail: zeyadz44@yahoo.com
} 
saprophytic, Listeria spp., Enterobacter spp., and Citrobacter spp., whereas four fungal pathogens were also identified in various food items. Among the fungal pathogens isolated were Aspergillus nigar (50\%), Candida krusei (19\%), Fusarium oxysporium (6\%) and Mucor (25\%). Antibiogram analysis reveals that Levofloxacin, Ceftriaxone Ciprofloxacin, Cefoxitin, Chloramphenicol, and Ceclor were more active against bacterial isolates. Fungal isolates had shown differential sensitivities toward Voriconazole. Local food markets of Peshawar and Mardan, KPK, Pakistan have more than 20\% risk of foodborne pathogens. It is recommended that the general public should purchase neat and clean food and adopt mild processing techniques to make the food hygienic.

Keywords: foodstuff, bacterial strain, fungal strain, antibacterial, antifungal

\section{Introduction}

The crucial constituents of the human body almost totally rely on food intake [1]. Surveillance of food management is a basic step toward food safety; in case it fails, foodborne infections can invade (via bacterial, fungal, viral and parasitic infections) and make many threats to health $[2,3]$. During the last few decades, in developing countries, food product, i.e., fruits, vegetables and canned foods have emerged as a new vehicle for the transmission of foodborne diseases associated with etiological agents. An increase in outbreaks has been reported all over the world $[4,5]$.

Among pathogenic microbes, bacteria are predominantly responsible for foodborne ailments, which often leads to outbreaks and increased death rates. Fluoroquinolone-resistant Campylobacter jejuni infections were found in raw milk [6]. Salmonella enterica has been found in fresh fruit, e.g., Salmonella braenderup in ready-to-eat mangoes, Salmonella Typhimurium and Salmonella Newport in cantaloupe, Salmonella agona in papayas and Salmonella newport in watermelon [7]. Pathogenic Escherichia coli O157:H7 and Listeria monocytogenes have been found in a delicatessen appetizer salad [8], ready-to-eat salads, organic spinach and romaine lettuce [9]. Bacillus subtilis and Mucor spp. revealed an extreme number of bacterial and fungal infections, although Chloramphenicol remain the effective agent for the controlling of bacterial microflora, as the exclusion of B. subtilis the degree variation in the antibiotic susceptibility were detected [10]. Fungal infection is particularly lethal, i.e., fungi produce dangerous and hazardous toxins and spores in foodstuffs, which lead to spoilage of food [11]. The detection of these toxins and spores is left behind an outbreak of food infection, as the diagnosis of the infection agents are exposed [12]. In particular, the fungal species are mostly found in the rotten tomato fruits comparatively more effective and potent than bacteria. Filamentous fungi usually produce various types of Aflatoxins and Mycotoxins [13].

Food contamination also leads to food poisoning, like diarrhea: which is fatal and may result in high death rates. The mechanism of the disease is the acquisition of toxins produced by microbes or may be due to the sensitivity of the host to the microbes itself [14]. For instance, the presence of Staphylococcus aureus in food is a great health problem for the community. Many of the staphylococcal species enormously produce heat-stable enterotoxins, which may result in gastroenteritis in humans. The main isolation source of the $C$. perfringes is raw meat, soil and intestinal tract of humans. Pathogeneses involve the production of toxins. Bacillus cereus mostly contaminated the fried rice, which accounts for the main source of the diseases, while such a sign of food illnesses has not been reported previously [14]. The spores produced by $B$. cereus resist heat, which were allowed to increase in huge numbers when the rice remains at night at room temperature prior to being fried. Different sets of rice were mixed; if these vanished then detection of $B$. cereus would be difficult in fried rice. The methicillin-resistant Staphylococcus aureus infections have been reported in the USA for up to 30 years $[5,15]$. Clostridium perfringens is usually found in raw meat, soil and the intestinal tract of humans $[16,17]$.

Raw vegetable and fruit cuts are generally sold on the roadside without any protective cover in Peshawar and Mardan. Nowadays the trend of selling and purchasing of ready-made food is increasing, including chickpea, beans, meat, chicken and fish on the roadside [18]. The local manufacturers are selling low-quality packed food without quality control evaluation by food authorities. The incidence of resistant bacteria in foodstuffs is remarkably important and has a wideranging ratio. The current investigation was to explore the frequency distribution of food-borne pathogenic bacteria and fungi. Furthermore, MRSA and MDRbacteria are found in food samples. Moreover, we studied the efficacy of differently available antibiotics evaluated to treat food-borne diseases.

\section{Material and Methods}

The current research study was carried out in the Microbiology Research Laboratory (MRL), Abasyn University Peshawar from April 2016 to March 2017. The work focused on isolation, identification and antibiotic profiling of pathogenic microbes from various food items. 


\section{Samples Collection}

About 200 samples of meat, chicken, fish, readymade and pack food were collected, and $25 \mathrm{~g}$ of each sample was excised using a sterile scalpel and put in a sterile test tube containing $6 \mathrm{ml}$ of buffered peptone water, followed by transportation to the laboratory within 1 hour. Collected food samples were processed immediately or preserved at $4^{\circ} \mathrm{C}$ in the refrigerator. Samples were cultured on nutrient agar.

\section{Culturing and Gram Staining}

The samples were investigated on Grams reaction followed by characterization by selective and differential media, e.g., MacConkey agar and blood agar.

\section{Biochemical Test}

The samples were characterized by using biochemical assays, i.e., triple sugar iron (TSI), urease test, citrate utilization tests, and indole test. Triple sugar iron agar medium was used for identifying Gramve enteric bacilli on the basis of dextrose, lactose and sucrose fermentation and $\mathrm{H}_{2} \mathrm{~S}$ production. The isolates were stabbed in the butt in a test tube with the help of a straight wire loop and streaked on the slope, followed by inoculation at $37^{\circ} \mathrm{C}$ for 24 hours. Indole test and then the specimen was inoculated with the help of a wire loop, then incubated at $37^{\circ} \mathrm{C}$ for 24 hours. After incubation at 24 hours, $0.5 \mathrm{ml}$ of Kovacs reagents were added in test tubes. Urease media $6 \mathrm{ml}$ sample of media was added in each test tube. Urea reagent was added to the media and then allowed to solidify, and then inoculum was streaked on it using sterilized inoculating wire loops. Citrate utilization test and the citrate agar media was prepared and the quantity of $6 \mathrm{ml}$ was poured into each test tube. Media was allowed to solidify and then inoculated with a straight wire loop and incubated at $37^{\circ} \mathrm{C}$ for 24 hours. Thereafter, test tubes were observed for bacterial growth. The test was based on the ability of an organism to use citrates as its only sources of carbon and ammonia as a source of nitrogen. If no color it was negative, if there was a change in the color it was considered positive. Catalase test for catalase test glass slide was cleaned and we placed a small drop of normal saline on the slide. With a sterilized and cooled inoculating loop we then picked up a small amount of the culture from the nutrient agar slant or Petri plate. The colonies were emulsified on each drop to make a smooth suspension. With a Pasteur pipette, we placed one drop of hydrogen peroxide over the test smear. And we observed the fluid over the smears for the appearance of gas bubbles for the positive results.

\section{Disc Diffusion Assay Bacteria}

Antibiotic sensitivity was conducted through the Kirby-Bauer disc diffusion technique following standard clinical laboratory standards institute (2015) guidelines. A panel of commonly prescribed antibiotics (Vancomycin, Azithromycin, Amoxicillin, Cefotaxime, Methicillin, Ceftriaxone, Levofloxacin, Cefixime, Cefuroxime, Ciprofloxacin, Cefoxitin, Chloramphenicol, Ceclor, and Linezolid) were used for the susceptibility of bacterial isolates. Briefly, 24 hours' fresh culture of various bacterial isolates were inoculated into each test tube containing, respectively, $8 \mathrm{ml}$ nutrient broth media. The culture tubes were incubated for 24 hours at $37^{\circ} \mathrm{C}$ in the bacterial culture. The next day, turbidity of the bacterial culture was adjusted with McFarland Solution. Uniform lawns for each isolate were prepared on nutrient agar media using sterilized cotton swabs. A panel of selected antibiotics was gently placed on the prepared lawns at an equal distance using sterilized forceps. All Petri plates were incubated for 24 hours at $37^{\circ} \mathrm{C}$, and the next day, zones of inhibitions created by antibiotics were measured.

\section{Isolation of Fungi}

A total of 100 randomly selected spoilt fruits and another 100 healthy looking fruits were examined. The fruits were cut into small segments (3 $\mathrm{mm}$ in diameter) with a sterilized blade, surface sterilized in $1 \%$ hypochlorite for $2 \mathrm{~min}$, plated on Sabouraud dextrose agar (SDA) aseptically and then incubated at $28^{\circ} \mathrm{C}$ for 5 days. A pure culture was obtained and maintained by sub-culturing each of the different colonies that emerged onto the SDA plates and were incubated at $28^{\circ} \mathrm{C}$ for 5 days. As a control, each of the healthy fruits was sterilized with $75 \%$ ethanol. The fruits were cut into small segments $(3 \mathrm{~mm}$ in diameter) with a sterile blade, placed on SDA and then incubated at $28^{\circ} \mathrm{C}$ for 5 days.

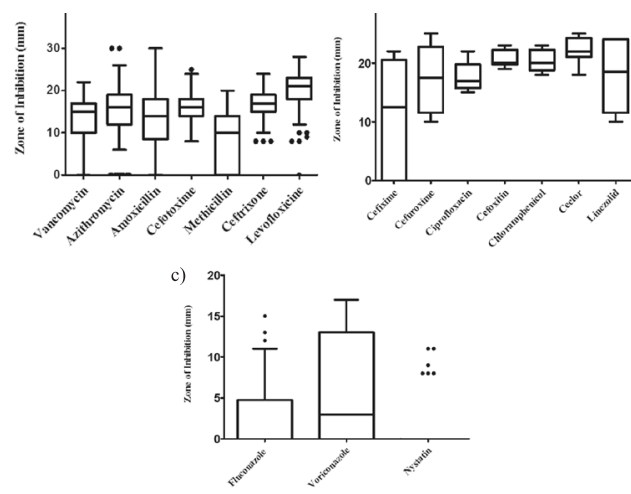

Fig. 1. Percentage frequency of bacterial positive and fungal positive samples among the total. 


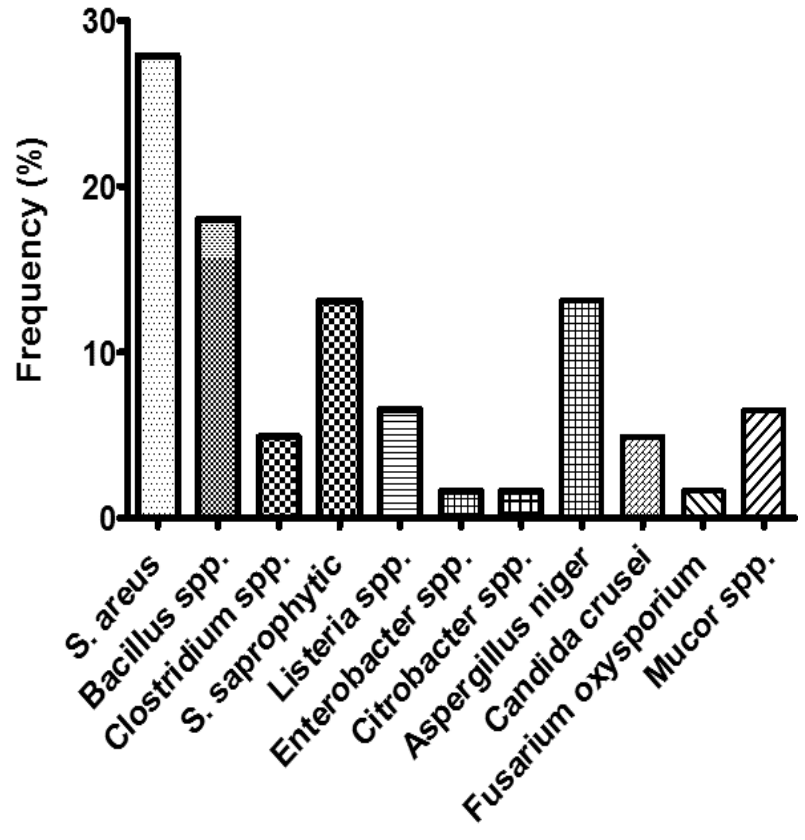

Fig. 2. Frequency distribution of bacterial and fungal isolates among the positive food samples.

\section{Identification Method for Fungi}

Agar disc diffusion method was used for screening antifungal activities of each antibiotic. Yeast inoculums in $0.85 \% \mathrm{NaCl}$ solution was spread on the surface of yeast extract-peptone-glycerol (YPG) agar plate \{Javed, $2016 \# 327\}$. Sterile filter paper discs (6mm in diameter) with $50 \mu \mathrm{g}$ of Nystatin, $25 \mu \mathrm{g}$ of Fluconazole, $1 \mu \mathrm{g}$ of Voriconazol and with $10 \mu \mathrm{l}$ of Caspofungin acetate in the concentration of $5 \mu \mathrm{g} / \mathrm{ml}$ were placed on the inoculated plates. Ultrapure water was used as negative control.

\section{Results}

In order to find out foodborne pathogenic bacteria and fungi in various foodstuffs, a total of 520 collected and evaluated food samples, $23.46 \%$ of the samples were found to be positive for different types of bacteria and fungi. Among the positive samples, the bacteria were predominant $(17.30 \%)$ as compared to fungi $(6.15 \%)$ (Fig. 1). The ratio of Gram-negative was higher than Gram-positive. Morphological characteristics, a)

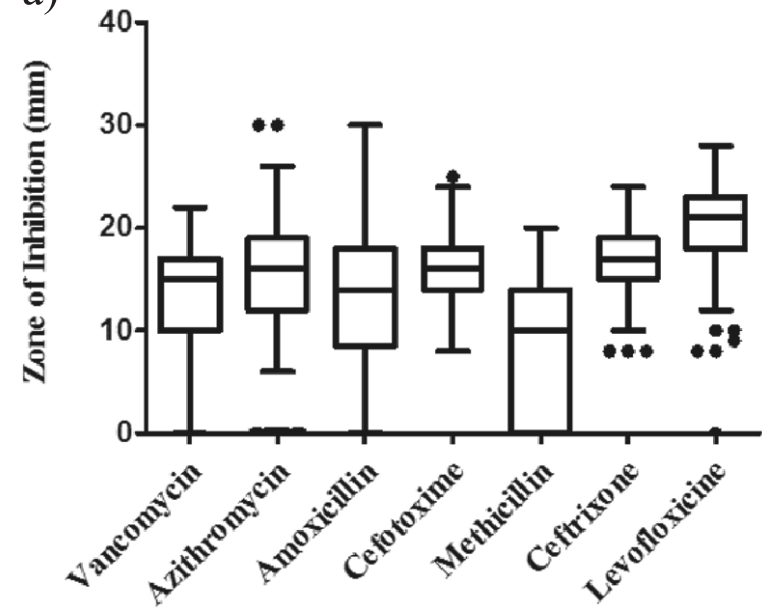

b)

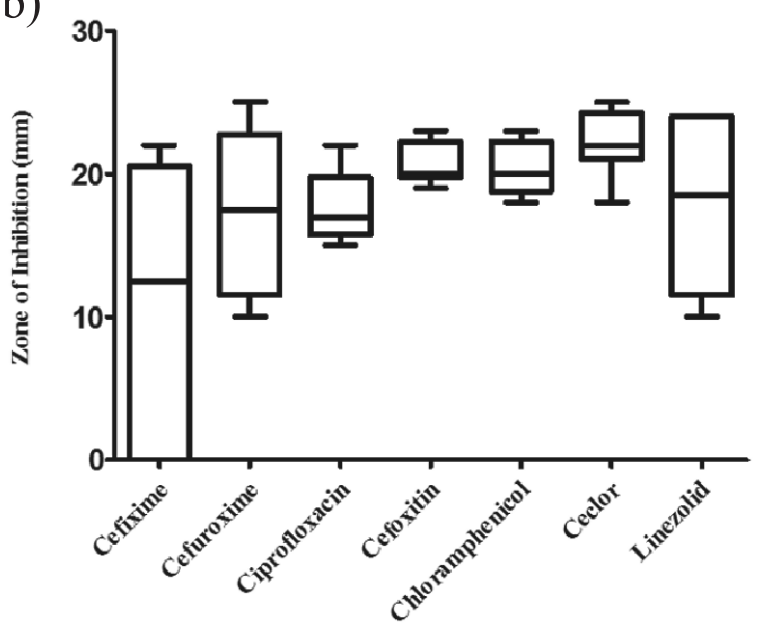

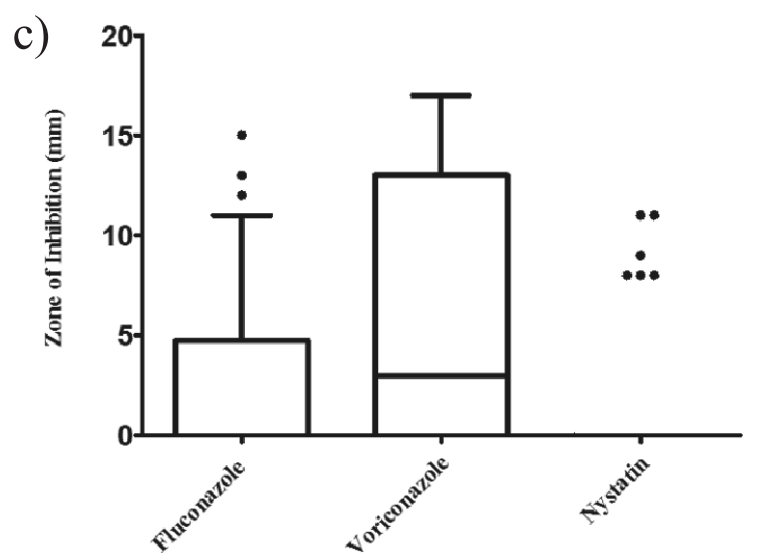

Fig. 3. Sensitivity profile of a) Gram positive, b) Gram negative and c) fungal isolates towards a panel of commonly used antibiotics and antifungals. 
a)

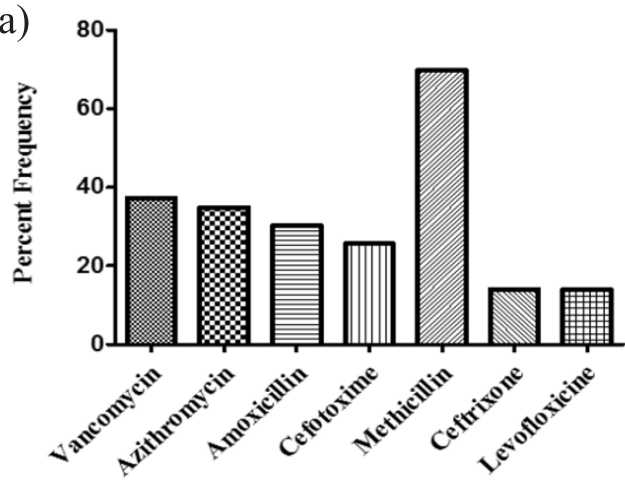

b)

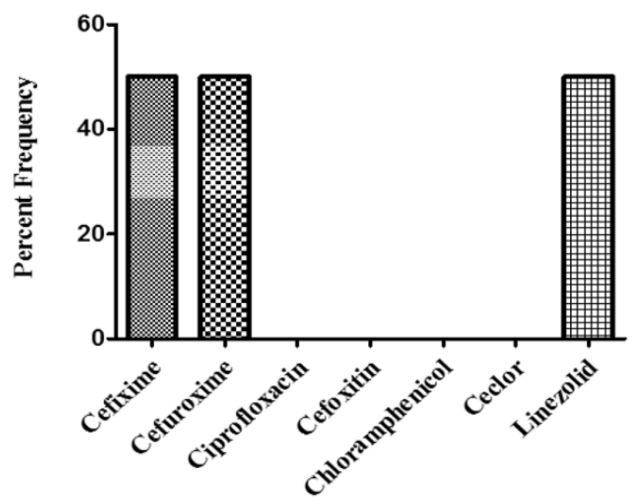

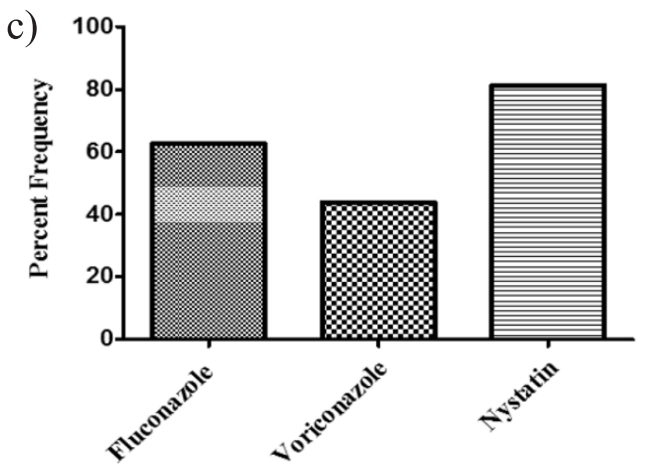

Fig. 4. Resistance profile of a) Gram positive, b) Gram negative and c) fungal isolates towards a panel of commonly used antibiotics and antifungals.

microscopic examination, and biochemical characterization revealed the presence of Staphylococcus aureus, Bacillus spp., Clostridium spp, Staphylococcus saprophytic, Listeria spp, Enterobacter spp., Citrobacter sp, Aspergillus niger, Candida crusei, Fusarium oxysporium and Mucor spp. (Fig. 2).

A panel of selected drugs was used to assess the susceptibility of pathogenic microbes. For the sensitivity of bacterial pathogens, a panel of antibiotics including Vancomycin, Azithromycin, Amoxicillin, Cefotaxime, Methicillin, Ceftriaxone, Levofloxacin, Cefixime, Cefuroxime, Ciprofloxacin, Cefoxitin, Chloramphenicol, Ceclor, and Linezolid was used. All the Gram-positive isolates had relatively similar and greater sensitivity towards Cefotaxime, Methicillin, and Levofloxacin as compared to the rest of the antibiotics (Fig. 3a). Similarly, Cefoxitin, Chloramphenicol, and Ceclor proved to be relatively more and equipotent towards the Gram-negative isolates (Fig. 3b). Among the antifungals, Voriconazole was found to be relatively more potent against the fungal isolates (Fig. 3c).

The percentage of frequencies of resistance microbial isolates were also determined (Fig. 4). Overall, the frequency of resistance was higher in Gram-positive as compared to that of Gram-negative (Fig. 4a and 4b). Frequencies of bacterial isolates showing resistance towards Methicillin, Cefixime, Ciprofloxacin, and Linezolid were comparatively high. Among the antifungals, the highest resistance was observed in the fungal isolates towards Nystatin (Fig. 4c).

\section{Discussion}

Foodborne diseases are very frequent and can easily be transmitted from contaminated food and food handlers. Furthermore, the incidence of resistant bacteria in foodstuffs is a worldwide phenomenon. It is a main community health risk $[19,20]$. At the same time, these organisms have been isolated from an extensive variety of foodstuffs consumed by man [21, 22]. Therefore, the current study was designed to evaluate various foods for the detection of bacterial and fungal contaminants and their susceptibility assessment towards the selected antibiotics and antifungals commonly available in the market.

Staphylococcus aureus, Bacillus spp., Clostridium spp., Staphylococcus saprophytic, Listeria spp., Enterobacter spp., and Citrobacter sp were identified among the bacteria in various kinds of food. Similar microbes have also been reported earlier from different sources of food $[19,20]$. Besides bacterial pathogens, fungal isolates Aspergillus niger, Candida crusei, Fusarium oxysporium and Mucor spp were also identified in various types of food. Similar fungal isolates were also discovered in different foods before [23-25].

For the susceptibility evaluation of microbes isolated from foods, panels of selected antibiotics and antifungals were used [26]. These include Vancomycin, Azithromycin, Amoxicillin, Cefotaxime, methicillin, Ceftriaxone, Levofloxacin (for Gram-positive bacteria) 
and for gram -veCefixime, Cefuroxime, Lanzolid, Cefoxitin, Ciprofloxacin, Ceclor, and Chloramphenicol (for Gram positive bacteria) were assessed for their antibacterial activities against the bacterial isolates. For fungal pathogens the antifungals Fluconazol, Voriconazol, and nystatin were used [27].

Gram-positive isolates were proved to be relatively more susceptible to Levofloxacin and Ceftriaxone. However, Vancomycin and methicillin were found to be relatively less active against the Gram positive isolates. These findings are in line with earlier reports [28, 29]. Ciprofloxacin, Cefoxitin, Chloramphenicol and ceclor have shown relatively highest activity against Gramnegative isolates. However, Cefixime displayed low potency against the Gram-negative isolates.

Besides bacterial isolates, four fungal pathogens were also identified in raw and ready-to-eat food. Three antifungal drugs - Fluconazol, Voriconazol, and Nystatin - were used for their potency against the isolated fungal pathogens. The Voriconazol was found to be comparatively more effective against all isolates. Low antifungal activity was observed for the Nystatin. Previous studies also show differential activities of various antifungals against the fungal isolates recovered from various foods $[23,30]$.

The resistance of microbial isolates to a drug was also determined in terms of percent isolates showing resistance to a specific drug. Relatively the highest resistance frequency was observed towards Methicillin as compared to other antibiotics used against Gram-positive isolates [5, 15]. A similar level of resistance was observed in Gram-negative bacterial isolates towards Cefixime, Cefuroxime, and Linezolid. However, Ciprofloxacin, Cefoxitin, Chloramphenicol and Ceclor proved to be highly active against Gramnegative isolates. Resistance to the third-generation Cephalosporin has also been described in Gramnegative isolates [31, 32]. Although Resistance towards all antifungals was observed in the fungal isolates, the frequency of fungal isolates showing resistance towards Nystatin was relatively high. Similar fungal isolates with differential susceptibility towards various antifungals have been reported before Ramesh et al. [33].

Our study concluded that the proportion of food samples harboring bacterial pathogens was much higher than that of harboring fungal pathogens. The antibiotic sensitivity pattern of various antibiotics used in the study reveals that Levofloxacin, Ceftriaxone Ciprofloxacin, Cefoxitin, Chloramphenicol, and Ceclor were found to be more active against bacterial isolates. Highest resistance was observed among the Gram-positive isolates towards Methicillin, whereas the relatively high proportion of Gram-negative isolates were identified showing resistance to Cefixime and Cefuroxime. Fungal isolates had shown differential sensitivities towards the antifungal used in the study.

\section{Conclusions}

Our study concludes that local food markets of Peshawar and Mardan, KPK, Pakistan have more than $20 \%$ risk of foodborne pathogens. It is recommended that the general public should purchase neat and clean food and adopt mild processing techniques to make the food hygienic. Furthermore, most of the commonly used antibiotics were found to be effective against bacterial isolates except for Methicillin, which shows antifungal differenatial activities.

\section{Acknowledgements}

The authors gratefully acknowledge the support received from Abaysn University Peshawar and the support from Higher Education Commission (HEC), Pakistan, under the Research Grant Programs (No. 212104/SRGP/R\&D/HEC/2018，21-182 0/S RG P/R\&D/ $\mathrm{HEC} / 2018$.

\section{Conflict of Interest}

The authors declare no conflict of interest.

\section{References}

1. SIMOPOULOS A.P. Evolutionary Aspects of the Dietary Omega-6/Omega-3 Fatty Acid Ratio: Medical Implications, in Evolutionary Thinking in Medicine. Springer. 119, 2016.

2. WEBB M., MORANCIE A. Food safety knowledge of foodservice workers at a university campus by education level, experience, and food safety training. Food Control. 50, 259, 2015.

3. MIKULSEN M., DIDUCK A.P. Towards an integrated approach to disaster management and food safety governance. International Journal of Disaster Risk Reduction. 15, 116, 2016.

4. HUSSAIN, A. Risk of Transmission of Antimicrobial Resistant Escherichia coli from Commercial Broiler and Free-Range Retail Chicken in India. Front Microbiol. 8, 2120, 2017.

5. WENDLANDT S., SCHWARZ S., SILLEY P. Methicillinresistant Staphylococcus aureus: a food-borne pathogen? Biomed Res Int. 4, 117, 2013.

6. SHEN C. Outbreak of Fluoroquinolone-Resistant Campylobacter jejuni Infections Associated with Raw Milk Consumption from a Herdshare Dairy - Colorado, 2016. Biomicrofluidics. 67 (5), 146, 2018.

7. GUO M., YADAV M.P., JIN T.Z. Antimicrobial edible coatings and films from micro-emulsions and their food applications. Biochemistry. 263, 9, 2017.

8. TSIRAKI M.I., et al., Viability of and Escherichia coli O157:H7 and Listeria monocytogenes in a delicatessen appetizer (yogurt-based) salad as affected by citrus extract $(\operatorname{Citrox}((\mathrm{c})))$ and storage temperature. Food Microbiol. 69, 11, 2018.

9. Control, C.f.D. and Prevention, Surveillance for foodborne disease outbreaks--United States, 2009-2010. MMWR. Morbidity and mortality weekly report. 62 (3), 41, 2013. 
10. PUNJA Z.K., RODRIGUEZ G., TIRAJOH A. Effects of Bacillus subtilis strain QST 713 and storage temperatures on post-harvest disease development on greenhouse tomatoes. Crop Protection. 84, 98, 2016.

11. AZIMAN N. Phytochemical profiles and antimicrobial activity of aromatic Malaysian herb extracts against foodborne pathogenic and food spoilage microorganisms. J Food Sci. 79 (4), M583, 2014.

12. TIWARI A.K. An Overview of Major Fungal Diseases of Sugarcane in India: Detection and Management Strategies, in Molecular Markers in Mycology. Springer. 275, 2017.

13. PATERSON R.R., LIMA N. Thermophilic Fungi to Dominate Aflatoxigenic/Mycotoxigenic Fungi on Food under Global Warming. Int J Environ Res Public Health. 14 (2), 2017.

14. MANNING L. Categorizing food-related illness: Have we got it right? Critical reviews in food science and nutrition. 57 (9), 1938, 2017.

15. ABDI R.D. Antimicrobial Resistance of Staphylococcus aureus Isolates from Dairy Cows and Genetic Diversity of Resistant Isolates. Foodborne Pathog Dis. 2018.

16. SAKANOUE H. Adherence of Clostridium perfringens spores to human intestinal epithelial Caco-2 cells. FEMS Microbiol Lett, 2018.

17. LEE C.A., LABBE R. Distribution of Enterotoxin- and Epsilon-Positive Clostridium perfringens Spores in U.S. Retail Spices. J Food Prot. 394, 2018.

18. JAVED A. Food Borne Health Issues and Their Relevance to Pakistani Society. American Scientific Research Journal for Engineering, Technology, and Sciences (ASRJETS). 26, (4), 235, 2016.

19. WANG H. Pathogenicity and antibiotic resistance of coagulase-negative staphylococci isolated from retailing chicken meat. LWT. 90, 152, 2018.

20. MISIHAIRABGWI J. Mycotoxin contamination of foods in southern africa: A 10-year review (2007-2016). Critical reviews in food science and nutrition. 1, 2017.

21. GHALY T.M. Evolution of class 1 integrons: Mobilization and dispersal via food-borne bacteria. PLoS One. 12 (6), e0179169, 2017.

22. JIN S.Q., YIN B.C., YE B.C. Multiplexed bead-based mesofluidic system for detection of food-borne pathogenic bacteria. Appl Environ Microbiol. 75 (21), 6647, 2009.
23. XIONG C. In vitro Antimicrobial Activities and Mechanism of 1-Octen-3-ol against Food-related Bacteria and Pathogenic Fungi. J Oleo Sci. 66 (9), 1041, 2017.

24. XING F. Recent developments and applications of hyperspectral imaging for rapid detection of mycotoxins and mycotoxigenic fungi in food products. Crit Rev Food Sci Nutr. 1, 2017.

25. PELTOMAA R. Species-specific optical genosensors for the detection of mycotoxigenic Fusarium fungi in food samples. Anal Chim Acta. 935, 231, 2016.

26. REN X., LIU W., LIU Y. Effects of fluconazole on the clinical outcome and immune response in fungal coinfected tuberculosis patients. Microb Pathog. 2018.

27. RAJKOWSKA K., KUNICKA-STYCZYŃSKA A., MAROSZYŃSKA M. Selected essential oils as antifungal agents against antibiotic-resistant Candida spp.: in vitro study on clinical and food-borne isolates. Microbial Drug Resistance. 23 (1), 18, 2017.

28. AGWA O.K., UZOIGWE C.I., WOKOMA E.C. Incidence and antibiotic sensitivity of Bacillus cereus isolated from ready to eat foods sold in some markets in Porth are court, Rivers State, Nigeria. Asian Journal of Microbiology, Biotechnology and Environmental Sciences. 14, 13, 2012.

29. TURUTOGLU H.U., ERCELIK S., OZTURK D. Antibiotic resistance of Staphylococcus aureus and coagulase-negative staphylococci isolated from bovine mastitis. Bulletin-Veterinary Institute In Pulawy. 1,50 (1), 41, 2006.

30. LYU A. Reveromycins A and B from Streptomyces sp. 3-10: Antifungal Activity against Plant Pathogenic Fungi In vitro and in a Strawberry Food Model System. Front Microbiol. 8, 550, 2017.

31. OLATOYE I.O., AMOSUN E.A., OGUNDIPE G.A.T. Multidrug-resistant Escherichia coli O157 contamination of beef and chicken in municipal abattoirs of southwest Nigeria. Nature and Science. 10 (8), 125, 2012.

32. PRIYADARSHINI K.L.R., RAMASWAMY R. Isolation and antibiotic sensitivity pattern of Citrobacter species with ESBL and AmpC detection at tertiary care hospital, Bangalore. J Evolution Med Dent Sci. 5 (30), 1553, 2016.

33. RAMESH S., KUMAR R.S., BALAJI R.M. Antibiotic susceptibility of Aspergillus spp. isolated from contaminated food sources. Der Pharmacia Lettre. 5 (6), 179, 2013. 
